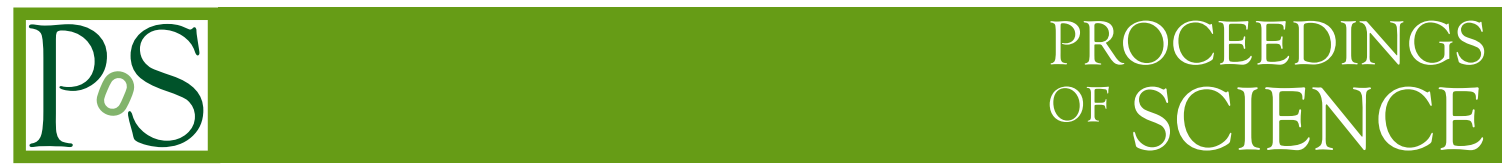

\title{
Recent progress in jet substructure calculations
}

\author{
Daniel Reichelt ${ }^{a * *}$ \\ ${ }^{a}$ Institut für Theoretische Physik, Georg-August-Universität Göttingen, \\ Friedrich-Hund-Platz 1, 37077 Göttingen, Germany \\ E-mail: daniel.reichelt@uni-goettingen.de
}

The substructure of QCD jets is an active area of research in both theory and experiment. This talk summarises the progress in calculations of jet substructure observables and the development of techniques to address the theoretical challenges posed by them. As particular example, the analysis of radiation inside jets using the Lund plane is reviewed. Fixed order and resummed calculations including the effect of the soft drop grooming technique are discussed for the observable family of jet angularities.

The Ninth Annual Conference on Large Hadron Collider Physics - LHCP2021

7-12 June 2021

Online

${ }^{*}$ Speaker 
Introduction. Since the start of the Large Hadron Collider (LHC), numerous experimental as well as theoretical studies have investigated, and significantly improved our understanding of, the distribution of radiation inside QCD jets. This is an important task, on the one hand to test and challenge our ability to accurately make QCD predictions, and on the other hand to find new ways to mitigate and suppress the background produced by QCD for example to searches for new physics. Here we will discuss some examples from the recent past, for a recent review aiming at a more inclusive picture see for example [1].

The Lund Plane. A useful representation of the QCD radiation phase space is the so called Lund plane [2]. An example is given in Fig. 1. The vertical axis represents a hardness measure like the transverse momentum $k_{t}$ of the radiation, while the horizontal axis marks the angular separation by some suitable measure $\Delta$, with the diagonal line in Fig. 1 representing the limit collinear to the jet axis. At leading logarithmic accuracy, QCD radiation is expected to be uniform in this plane. It has been used since its introduction until today for the development of parton showers $[3,4]$ and resummed calculations [5]. Non-trivial effects beyond the leading logarithms are associated with

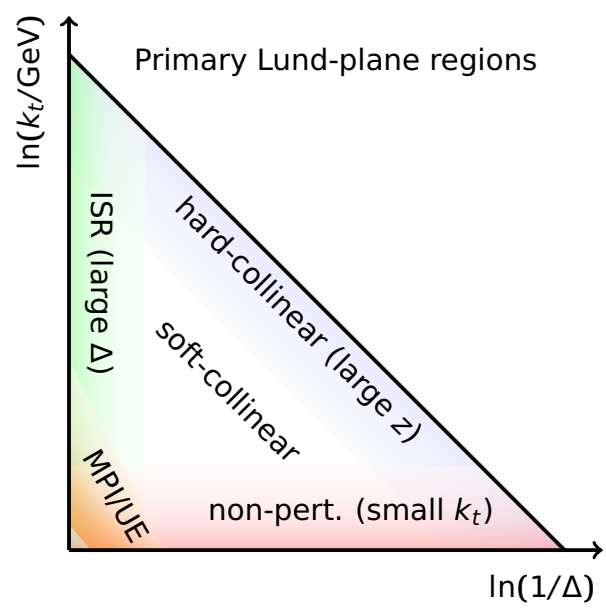

Figure 1: Primary regions of the Lund plane. Figure taken from [6]

different regions in the Lund plane. At larger $k_{t}$ the physics is dominated by perturbative effects that would usually be taken into account, in addition to fixed order calculations, by resummed expressions or parton shower simulations. At smaller transverse momenta, non-perturbative effects take over. A recent development is the utilisation of the Lund plane in measurements. This was proposed in [6] with concrete calculations carried out in [7]. Lund plane representations of a jet can be obtained by declustering the jet constituents with the Cambridge/Aachen algorithm. This measurement prescription has been realised by the LHC experiments $[8,9]$. The insights into the physical origin of radiation in different areas of the Lund plane can be used to construct optimal observables for signal and background discrimination for Standard Model processes [10].

Soft Drop grooming. The contributions at smaller $k_{t}$ scales, like hadronisation and the underlying event (UE), can not be calculated from first principles but need to be modelled. A variety of tools is available to reduce the impact of such corrections to jet observables. The example that shall be examined here is the soft drop grooming procedure [11]. Similar to the measurement prescription 
of the Lund plane, a jet with radius $R$ is declustered with the Cambridge/Aachen algorithm. The last splitting is undone and it is checked if the two sub-jets $i, j$ satisfy the soft drop condition

$$
z_{g}=\frac{\min \left(p_{T, i}, p_{T, j}\right)}{p_{T, i}+p_{T, j}}>z_{\mathrm{cut}}\left(\frac{\Delta R_{i j}}{R}\right)^{\beta}
$$

where $p_{T, i}$ is the transverse momentum of jet $i$ and $\Delta R$ is the usual separation between $i$ and $j$ in the plane of rapidity $y$ and azimuth $\phi, \Delta R_{i j}^{2}=\left(y_{i}-y_{j}\right)^{2}+\left(\phi_{i}-\phi_{j}\right)^{2}$. The effect of the groomer can be adjusted with the parameters $z_{\text {cut }}$ and $\beta$. The focus here will be on the grooming mode $\beta \geq 0$. In terms of the emission of soft gluons from a jet, soft drop cuts out precisely the soft wide-angle corner of the phase space marked as "UE/MPI" in Fig. 1. Analytical understanding of the behaviour of this groomer can be gained by examining the $p_{T}$ fraction $z_{g}$ and the angular separation $\theta_{g}=\Delta R_{i j} / R$ of the two sub-jets that satisfy the criterion in Eq. (1). These obervables were first analysed in [11, 12], and calculated at next-to-leading logarithmic accuracy in [13, 14].

With the sensitivity of an object to non-perturbative effects reduced, it is possible to calculate for example jet-shape type observables from the constituents that survive grooming. This principle can be extended to event shapes in electron-positron [15, 16] and proton-proton [17] collisions. The resulting observables can feature an impressively large range where the perturbative calculations are dominated by the logarithmic behaviour, but non-perturbative effects are still negligible. This has been applied to the groomed jet mass, which was measured by the ATLAS [18] and CMS [19] experiments, and successfully compared to calculations at various accuracies [20-23].

Jet Angularities An example of a jet observable that shall be examined closer here is the family of jet angularities, defined on the constituents of a jet $J$, that might have been subject to the soft drop grooming procedure described above, by

$$
\lambda_{\alpha}^{\kappa}=\sum_{i \in J}\left(\frac{p_{T, i}}{p_{T, J}}\right)^{\kappa}\left(\frac{\Delta R_{i}}{R}\right)^{\alpha} .
$$

Here $\Delta R_{i}$ denotes the angular distance of the jet constituent $i$ with respect to the jet axis. In the case where $\alpha \leq 1$, it is convenient to choose this to be the winner-takes-all axis. Several values of $\kappa$ are conventionally studied experimentally, but since out of these choices only $\kappa=1$ is infrared safe, the discussion here will be restricted to this choice. Angularities are among the standard observables considered in the context of quark-gluon discrimination studies in recent years [24-28] that have been a driver for developments in theory and modelling [29-32]. Recent studies have also considered tagging initial state flavours using angularities of final state jets [27, 33]. Common choices for $\alpha$ are $\alpha \in\{0.5,1,2\}$. Measurements at the LHC are presented for example in [34-36]. Theoretical predictions are available in a variety of frameworks [37-43]. Calculations are also performed for related observables at lepton colliders [5, 44, 45] and in deep inelastic scattering $[46,47]$. A recent calculation is the one presented in [43], closely following the precise measurement prescription in [36] for the groomed and ungroomed angularities measured on the leading anti- $k_{t}$ jet in $Z+$ jet production. It is based on the CAESAR formalism [5] for NLL resummation and uses the automated implementation [48, 49] available within the SHERPA [50, 51] framework. The extension to soft drop groomed observables was introduced in [17]. The fixed order calculation at NLO accuracy is performed using COMIX [52] in conjunction with OPENLOOPS [53] and RECOLA 

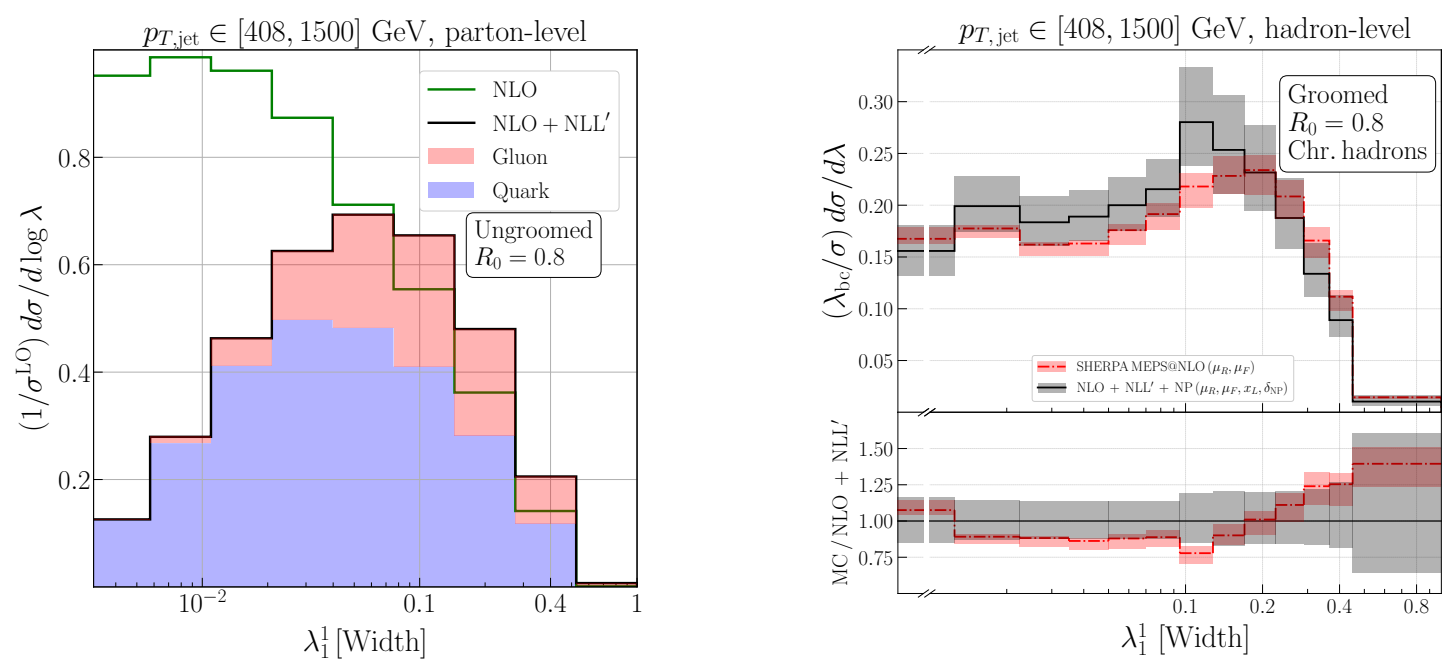

Figure 2: Jet angularity with $\kappa=1$ and $\alpha=1$ of the leading jet in $p p \rightarrow Z+$ jet production at $\sqrt{s}=13 \mathrm{TeV}$. Left: Perturbative prediction at NLO (green) and NLO+NLL' (black) accuracies for ungroomed jets. The face colours illustrate the contributions for gluon (red) and quark (blue) jets. Right: Predictions for groomed jets at NLO+NLL' (black) including non-perturbative corrections obtained from Monte Carlo simulations, compared to the distribution obtained from SHERPA at MEPS@NLO. Figures are taken from [43].

[54, 55]. A flavour sensitive matching scheme [17, 56] allows to achieve overall NLO+NLL' accuracy. The dependence of the soft wide-angle contribution proportional to powers of the jet radius $R$ is accounted for using expressions taken from [57], and non-global logarithms are computed numerically with the algorithm first introduced in [58].

The left plot in Fig. 2 displays this result for the $\alpha=1$ angularity, together with the NLO fixed order prediction. The effect of including the resummation is visible over the full range of the observable. This is related to the large LO corrections to the cross section. For reference, it is illustrated how the quark and gluon jet contributions stack up to the full matched result. While the large angularity region receives a significant contribution from gluon jets, the soft tail of the distribution is entirely dominated by quark jets. The right hand plot in Fig. 2 compares the matched result to a parton shower prediction obtained from SHERPA at MEPS@NLO accuracy [59], again for the $\alpha=1$ angularity but including the effect of grooming. Non-perturbative corrections are obtained as averaged ratios of the parton- and hadron-level predictions of SHERPA as well as HERWIG [60, 61] and PYTHIA [62]. Within uncertainties, parton shower and resummation show the same behaviour in this example. This statement is however dependent on the choice of $\alpha$, in particular in regions where shower cutoff effects become important.

Conclusion The substructure of jets in hadron production is an active field of research with an interesting interplay between experiment, theory, the developments of analysis methods and modelling in Monte Carlo generators. Both resummed calculations as well as parton shower simulations are crucial to the current understanding. Studying their relation can be expected to play an important role in the future [63-65]. In terms of analytic calculations, a higher order understanding of non-global logarithms [66] as well as transition point effects in the case of groomed observables [67] appears desirable. 
Acknowledgments I am grateful to Steffen Schumann for his comments on the original talk and this manuscript. I acknowledge funding from the European Union's Horizon 2020 research and innovation programme as part of the Marie Skłodowska-Curie Innovative Training Network MCnetITN3 (grant agreement no. 722104), from BMBF (contract 05H18MGCA1) and by the Deutsche Forschungsgemeinschaft (DFG, German Research Foundation) - project number 456104544.

\section{References}

[1] A. J. Larkoski, I. Moult, and B. Nachman, Jet Substructure at the Large Hadron Collider: A Review of Recent Advances in Theory and Machine Learning, Phys. Rept. 841 (2020) 1-63, [arXiv: 1709.04464].

[2] B. Andersson, G. Gustafson, L. Lonnblad, and U. Pettersson, Coherence Effects in Deep Inelastic Scattering, Z. Phys. C $\mathbf{4 3}$ (1989) 625.

[3] G. Gustafson, Multiplicity distributions in QCD cascades, Nucl. Phys. B 392 (1993) 251-280.

[4] K. Hamilton, R. Medves, G. P. Salam, L. Scyboz, and G. Soyez, Colour and logarithmic accuracy in final-state parton showers, JHEP $\mathbf{0 3}$ (2021) 041, [arXiv: 2011 . 10054].

[5] A. Banfi, G. P. Salam, and G. Zanderighi, Principles of general final-state resummation and automated implementation, JHEP 03 (2005) 073, [hep-ph/0407286].

[6] F. A. Dreyer, G. P. Salam, and G. Soyez, The Lund Jet Plane, JHEP 12 (2018) 064, [arXiv: 1807.04758].

[7] A. Lifson, G. P. Salam, and G. Soyez, Calculating the primary Lund Jet Plane density, JHEP 10 (2020) 170, [arXiv: 2007.06578$]$.

[8] ATLAS Collaboration, G. Aad et al., Measurement of the Lund Jet Plane Using Charged Particles in $13 \mathrm{TeV}$ Proton-Proton Collisions with the ATLAS Detector, Phys. Rev. Lett. 124 (2020), no. 22 222002, [arXiv: 2004 .03540].

[9] ALICE Collaboration Collaboration, Physics Preliminary Summary: Measurement of the primary Lund plane density in pp collisions at $\sqrt{s}=13 \mathrm{TeV}$ with ALICE, .

[10] C. K. Khosa and S. Marzani, Higgs boson tagging with the Lund jet plane, Phys. Rev. D 104 (2021), no. 5 055043, [arXiv:2105.03989].

[11] A. J. Larkoski, S. Marzani, G. Soyez, and J. Thaler, Soft Drop, JHEP 05 (2014) 146, [arXiv: 1402.2657].

[12] A. J. Larkoski, S. Marzani, and J. Thaler, Sudakov Safety in Perturbative QCD, Phys. Rev. D 91 (2015), no. 11 111501, [arXiv: 1502.01719].

[13] Z.-B. Kang, K. Lee, X. Liu, D. Neill, and F. Ringer, The soft drop groomed jet radius at NLL, JHEP 02 (2020) 054, [arXiv: 1908. 01783]. 
[14] P. Cal, K. Lee, F. Ringer, and W. J. Waalewijn, The soft drop momentum sharing fraction $z_{g}$ beyond leading-logarithmic accuracy, arXiv:2106.04589.

[15] J. Baron, S. Marzani, and V. Theeuwes, Soft-Drop Thrust, JHEP 08 (2018) 105, [arXiv: 1803.04719]. [Erratum: JHEP 05, 056 (2019)].

[16] S. Marzani, D. Reichelt, S. Schumann, G. Soyez, and V. Theeuwes, Fitting the Strong Coupling Constant with Soft-Drop Thrust, JHEP 11 (2019) 179, [arXiv: 1906. 10504].

[17] J. Baron, D. Reichelt, S. Schumann, N. Schwanemann, and V. Theeuwes, Soft-drop grooming for hadronic event shapes, JHEP 07 (2021) 142, [arXiv : 2012 . 09574].

[18] ATLAS Collaboration, M. Aaboud et al., Measurement of the Soft-Drop Jet Mass in pp Collisions at $\sqrt{s}=13 \mathrm{TeV}$ with the ATLAS Detector, Phys. Rev. Lett. 121 (2018), no. 9 092001, [arXiv: 1711.08341].

[19] CMS Collaboration, A. M. Sirunyan et al., Measurements of the differential jet cross section as a function of the jet mass in dijet events from proton-proton collisions at $\sqrt{s}=13 \mathrm{TeV}$, JHEP 11 (2018) 113, [arXiv: 1807.05974$].$

[20] C. Frye, A. J. Larkoski, M. D. Schwartz, and K. Yan, Factorization for groomed jet substructure beyond the next-to-leading logarithm, JHEP 07 (2016) 064, [arXiv: 1603.09338].

[21] C. Frye, A. J. Larkoski, M. D. Schwartz, and K. Yan, Precision physics with pile-up insensitive observables, arXiv: 1603.06375.

[22] S. Marzani, L. Schunk, and G. Soyez, The jet mass distribution after Soft Drop, Eur. Phys. J. C 78 (2018), no. 2 96, [arXiv: 1712 . 05105].

[23] S. Marzani, L. Schunk, and G. Soyez, A study of jet mass distributions with grooming, JHEP 07 (2017) 132, [arXiv: 1704.02210].

[24] A. J. Larkoski, J. Thaler, and W. J. Waalewijn, Gaining (Mutual) Information about Quark/Gluon Discrimination, JHEP 11 (2014) 129, [arXiv: 1408 . 3122].

[25] J. R. Andersen et al., Les Houches 2015: Physics at TeV Colliders Standard Model Working Group Report, in 9th Les Houches Workshop on Physics at TeV Colliders, 5, 2016. arXiv: 1605.04692.

[26] Les Houches 2017: Physics at TeV Colliders Standard Model Working Group Report, 3, 2018.

[27] S. Amoroso et al., Les Houches 2019: Physics at TeV Colliders: Standard Model Working Group Report, in 11th Les Houches Workshop on Physics at TeV Colliders: PhysTeV Les Houches, 3, 2020. arXiv:2003.01700. 
[28] P. Gras, S. Höche, D. Kar, A. Larkoski, L. Lönnblad, S. Plätzer, A. Siódmok, P. Skands, G. Soyez, and J. Thaler, Systematics of quark/gluon tagging, JHEP 07 (2017) 091, [arXiv: 1704.03878].

[29] J. Mo, F. J. Tackmann, and W. J. Waalewijn, A case study of quark-gluon discrimination at NNLL' in comparison to parton showers, Eur. Phys. J. C 77 (2017), no. 11 770, [arXiv: 1708.00867].

[30] D. Reichelt, P. Richardson, and A. Siódmok, Colour Reconnections in Quark and Gluon Jets in Herwig 7, Acta Phys. Polon. B 48 (2017) 1167-1173.

[31] D. Reichelt, P. Richardson, and A. Siodmok, Improving the Simulation of Quark and Gluon Jets with Herwig 7, Eur. Phys. J. C 77 (2017), no. 12 876, [arXiv: 1708.01491].

[32] A. J. Larkoski and E. M. Metodiev, A Theory of Quark vs. Gluon Discrimination, JHEP 10 (2019) 014, [arXiv: 1906.01639].

[33] S. Caletti, O. Fedkevych, S. Marzani, and D. Reichelt, Tagging the initial-state gluon, Eur. Phys. J. C 81 (2021), no. 9 844, [arXiv:2108.10024].

[34] ATLAS Collaboration, M. Aaboud et al., Measurement of jet-substructure observables in top quark, $W$ boson and light jet production in proton-proton collisions at $\sqrt{s}=13 \mathrm{TeV}$ with the ATLAS detector, JHEP 08 (2019) 033, [arXiv : 1903.02942].

[35] ALICE Collaboration, S. Acharya et al., Measurements of the groomed and ungroomed jet angularities in pp collisions at $\sqrt{s}=5.02 \mathrm{TeV}$, arXiv:2107.11303.

[36] CMS Collaboration, A. Tumasyan et al., Study of quark and gluon jet substructure in Z+jet and dijet events from pp collisions, arXiv: 2109.03340.

[37] S. D. Ellis, C. K. Vermilion, J. R. Walsh, A. Hornig, and C. Lee, Jet Shapes and Jet Algorithms in SCET, JHEP 11 (2010) 101, [arXiv: 1001.0014].

[38] A. Hornig, Y. Makris, and T. Mehen, Jet Shapes in Dijet Events at the LHC in SCET, JHEP 04 (2016) 097, [arXiv: 1601.01319].

[39] Z.-B. Kang, K. Lee, and F. Ringer, Jet angularity measurements for single inclusive jet production, JHEP 04 (2018) 110, [arXiv: 1801.00790].

[40] Z.-B. Kang, K. Lee, X. Liu, and F. Ringer, Soft drop groomed jet angularities at the LHC, Phys. Lett. B 793 (2019) 41-47, [arXiv: 1811. 06983].

[41] A. J. Larkoski, G. P. Salam, and J. Thaler, Energy Correlation Functions for Jet Substructure, JHEP 06 (2013) 108, [arXiv: 1305 . 0007].

[42] A. J. Larkoski, D. Neill, and J. Thaler, Jet Shapes with the Broadening Axis, JHEP 04 (2014) 017, [arXiv: 1401.2158]. 
[43] S. Caletti, O. Fedkevych, S. Marzani, D. Reichelt, S. Schumann, G. Soyez, and V. Theeuwes, Jet angularities in Z+jet production at the LHC, JHEP 07 (2021) 076, [arXiv:2104.06920].

[44] C. F. Berger, T. Kucs, and G. F. Sterman, Interjet energy flow / event shape correlations, Int. J. Mod. Phys. A 18 (2003) 4159-4168, [hep-ph/0212343].

[45] C. F. Berger, T. Kucs, and G. F. Sterman, Event shape / energy flow correlations, Phys. Rev. D 68 (2003) 014012, [hep-ph/0303051].

[46] E.-C. Aschenauer, K. Lee, B. S. Page, and F. Ringer, Jet angularities in photoproduction at the Electron-Ion Collider, Phys. Rev. D 101 (2020), no. 5 054028, [arXiv: 1910.11460].

[47] D. Kang, T. Maji, and J. Zhu, Angularity in DIS at next-to-next-to-leading log accuracy, arXiv:2106.14429.

[48] E. Gerwick, S. Hoeche, S. Marzani, and S. Schumann, Soft evolution of multi-jet final states, JHEP 02 (2015) 106, [arXiv: 1411.7325].

[49] N. Baberuxki, C. T. Preuss, D. Reichelt, and S. Schumann, Resummed predictions for jet-resolution scales in multijet production in $e^{+} e^{-}$annihilation, JHEP 04 (2020) 112, [arXiv: 1912.09396].

[50] T. Gleisberg, S. Hoeche, F. Krauss, M. Schonherr, S. Schumann, F. Siegert, and J. Winter, Event generation with SHERPA 1.1, JHEP 02 (2009) 007, [arXiv: 0811 . 4622].

[51] Sherpa Collaboration, E. Bothmann et al., Event Generation with Sherpa 2.2, SciPost Phys. 7 (2019), no. 3 034, [arXiv: 1905 .09127].

[52] T. Gleisberg and S. Hoeche, Comix, a new matrix element generator, JHEP 12 (2008) 039, [arXiv: 0808.3674].

[53] F. Buccioni, J.-N. Lang, J. M. Lindert, P. Maierhöfer, S. Pozzorini, H. Zhang, and M. F. Zoller, OpenLoops 2, Eur. Phys. J. C 79 (2019), no. 10 866, [arXiv: 1907. 13071].

[54] S. Actis, A. Denner, L. Hofer, J.-N. Lang, A. Scharf, and S. Uccirati, RECOLA: REcursive Computation of One-Loop Amplitudes, Comput. Phys. Commun. 214 (2017) 140-173, [arXiv: 1605.01090].

[55] B. Biedermann, S. Bräuer, A. Denner, M. Pellen, S. Schumann, and J. M. Thompson, Automation of NLO QCD and EW corrections with Sherpa and Recola, Eur. Phys. J. C 77 (2017) 492, [arXiv: 1704.05783].

[56] A. Banfi, G. P. Salam, and G. Zanderighi, Infrared safe definition of jet flavor, Eur. Phys. J. C 47 (2006) 113-124, [hep-ph/0601139].

[57] M. Dasgupta, K. Khelifa-Kerfa, S. Marzani, and M. Spannowsky, On jet mass distributions in Z+jet and dijet processes at the LHC, JHEP 10 (2012) 126, [arXiv: 1207 . 1640]. 
[58] M. Dasgupta and G. P. Salam, Resummation of nonglobal QCD observables, Phys. Lett. B 512 (2001) 323-330, [hep-ph/0104277].

[59] S. Hoeche, F. Krauss, M. Schonherr, and F. Siegert, QCD matrix elements + parton showers: The NLO case, JHEP 04 (2013) 027, [arXiv: 1207 . 5030].

[60] J. Bellm et al., Herwig 7.0/Herwig++ 3.0 release note, Eur. Phys. J. C 76 (2016), no. 4 196, [arXiv: 1512.01178].

[61] J. Bellm et al., Herwig 7.2 release note, Eur. Phys. J. C 80 (2020), no. 5 452, [arXiv: 1912.06509].

[62] T. Sjöstrand, S. Ask, J. R. Christiansen, R. Corke, N. Desai, P. Ilten, S. Mrenna, S. Prestel, C. O. Rasmussen, and P. Z. Skands, An introduction to PYTHIA 8.2, Comput. Phys. Commun. 191 (2015) 159-177, [arXiv: 1410. 3012].

[63] S. Höche, D. Reichelt, and F. Siegert, Momentum conservation and unitarity in parton showers and NLL resummation, JHEP 01 (2018) 118, [arXiv: 1711.03497].

[64] M. Dasgupta, F. A. Dreyer, K. Hamilton, P. F. Monni, and G. P. Salam, Logarithmic accuracy of parton showers: a fixed-order study, JHEP 09 (2018) 033, [arXiv : 1805 . 09327]. [Erratum: JHEP 03, 083 (2020)].

[65] M. Dasgupta, F. A. Dreyer, K. Hamilton, P. F. Monni, G. P. Salam, and G. Soyez, Parton showers beyond leading logarithmic accuracy, Phys. Rev. Lett. 125 (2020), no. 5 052002, [arXiv: 2002 . 11114].

[66] A. Banfi, F. A. Dreyer, and P. F. Monni, Next-to-leading non-global logarithms in QCD, arXiv:2104.06416.

[67] K. Benkendorfer and A. J. Larkoski, Grooming at the Cusp: All-Orders Predictions for the Transition Region of Jet Groomers, arXiv : 2108.02779. 